\title{
A Staffing Tool to Improve Efficiency at a Nursing Department
}

\author{
M.A.Louly ${ }^{\mathrm{a}}$, A. Gharbi ${ }^{\mathrm{a}}$, M. N. Azaiez ${ }^{\mathrm{b}}$, A. Bouras ${ }^{\mathrm{a}}$, Aymanmostafa ${ }^{\mathrm{a}}$, \\ Abdelatysayed, \\ ${ }^{A}$ kingsauduniversity, College Of Engineering, Industrial Engineering Department, P.O. Box 800, Riyadh, Saudi \\ Arabia, \\ ${ }^{B}$ university of Tunis, Tunis Business School, Al Mourouj, Tunisia
}

\begin{abstract}
The paper suggests a staffing tool to improve efficiency at a nursing department of a local hospital. The managers consider they are understaffed and try to overwhelm the staffing deficit problem through overtime, rather than hiring additional nurses. The estimates indicate that the shortage at the hospital level corresponds to 300 full time equivalent (FTE) nurses. However, the huge amount of allocated budget for overtime becomes a concern since the deficit is not accurately estimated. Indeed, the suggested staffing tool shows that some nursing units are unnecessarily overstaffed. Moreover, the current study reveals that the real deficit is of only215 FTEresulting in a potential saving of $28 \%$.
\end{abstract}

Keywords: nurse staffing, efficiency improvement, overstaffing, understaffing.

\section{LITERATURE REVIEW}

In most of the publications tackling the nurse staffing problem, researchers focus on studying the connection between the staffing level and the corresponding patient outcomes. Aiken et al. [1] report that increased specialized nursing staff contributes to lowering in-patient mortality rates as well as length of stay (LOS). Mchugh et al. [2] and Schwab et al. [3] go in the same direction by considering other outcomes like reducing poor glycemic control complications due to an increase in nurse staffing level. In particular, Schwab et al. [3] address understaffing (low nurse/patient ratio) with an emphasis on risk factors that cause nosocomial infections. Van den Heede et al. [4] come out with similar conclusions as in [3] since they recommend to evaluate the link between nurse staffing and ten patient outcomes potentially sensitive to nursingcare. Similarly, Needleman et al. [5] consider the relationship between the nurse staffing level and the hospitalized patients complication. Tubbs-Cooley et al. [6] conduct a study in the pediatric care to investigate the relationship between staffing ratios and all-causes of readmission among children admitted for common medical and surgical conditions.

McGahan et al [7] review the literature to investigate how nurse staffing affects mortality and morbidity in an adult Intensive Care Unit. Akinci and Krolikowski[8] conclude on the negative effect of nurse understaffing levels on the quality of care. As in [8], Ball et al. [9] report a fact from English hospitals where nurses fail to assure care due to time pressure-'missed care'. In the same topic, Kalisch et al. [10] analyze whether missed nursing care can be projected by actual nurse staffing. On the other hand, Clarke [11] criticizes researches on staffing as they "are not able to rule out all competing causes of the staffing/outcomes relationship". Moreover, he reports that low staffing levels are responsible for generating poor outcomes. This conclusion is enforced by Savitz et al. [12] who also require more consensus on potential measures that help in explaining how received quality of care is impacted by staffing changes.

From an economic point of view, Goryakin et al. [13] point out some issues in assessing the costeffectiveness of nurse staffing since it is associated with better outcomes and more expensive care. Nurse-scheduling can provide adequate staffing overtime accounting for demand fluctuations. Azaiez and Al Sherif[14] provide an optimized nursescheduling where patient care demand is variable. Their model meets the staffing requirements and show good potential for considerable savings by reducing overtime.

Maenhout and Vanhoucke[15] report that hospital operational costs is also impacted by management of nursing personnel. They state that "policy decisions on the staffing level have an impact on the outcome of the scheduling level and vice versa". 


\section{BACKGROUND}

The hospital faces an estimated shortage of 300 Full Time Equivalent (FTE) nurses according to the nursing department estimates. Given the relatively low wages of the nurses, the management adopted the policy of substituting these 300 FTE vacancies by allowing additional load to the existing nurses. These extra loads result in an overtime cost at the rate of 1.1 times the regular rate. On the one hand, this option is considered as a retention factor. In fact, it may substantially increase nurses' returns. On the other hand and compared to the various costs of recruiting more nurses (files processing, interviews, salaries, housing, airplane tickets, paid vacation leaves, etc.), overtime is by far less costly to the hospital. The budgetallocatedto overtimea mounts to $\$ 11.5$ million. The top management of the hospital considers this budget to be excessive. Another potential negative impact of this substitution strategy may be the risk of yield ing poor nursing services due to substantial overload caused by the excessive assigned overtime on most of the nurses.

\section{THE PROBLEM}

The study focuses on evaluating the current estimated staffing shortage of 300 (FTE) nurses. There is a concern of the top management that the hospital may be using "excessive" overtime to substitute for this staffing shortage. It also seems to be more efficient to keep with such a staffing shortage given the fixed and variable cost in the recruitment of nurses on one hand. And on the other hand, the overtime employed to meet the staffing requirements is also used as a retention factor to attract nursing staff by presenting them with additional means to add to their income, thus presenting the hospital with a competitive recruitment edge, given the relatively lower wages being offered to nurses. However, it is not clear to the top management whether the current staffing shortage amply justifies the $\$ 11.5$ million of overtime budget. Moreover, one may argue on whether the 300 FTE level represents the right level of staffing shortage. Consequently, this work deals with investigating this level and determining the required overtime on the basis of the hospital needs.

\section{DATA COLLECTION}

Data for each of the 32 in-patient units of the hospital was provided for the number of patients per unit, the budgeted NHPPD per unit, the actual six-week schedule of the study, and the corresponding total overtime per unit. Additional unreliable data was provided (budgeted productive hours, actual productive hours, actual NHPPD). The study has analyzed the additional data and detected many related problems. Then, the needed data was accurately calculated directly from the actual schedules. This was a tedious and time-consuming task. However, it provided some highly reliable inputs. Exchanges of information and validation of the calculated estimates have been made with the staffing department. The nursing leadership has shown full satisfaction with the new obtained estimates.

The number of nurses amounts to 1,114 nurse. The used number of hours over the six-week schedule under investigation was 261,308 hour. The applied overtime was of 46,270 hour.

\section{DATA PROCESSING AND RESULTS}

After processing the provided data, the following key elements have been calculated. The used definitions are presented in this section along with the calculations made on the data of the studied 6-week period.

At the aggregate level, there are 1114 available nurses acting for direct patient care in the 32 units. Each nurse is supposed to work 22 shifts in a 6-week period. The duration of each shift is 12 hours.

- At the aggregate level, the available regular productive hours are deduced from the total productive hours and the total Overtime as follows:

Used hours - Overtime hours $=$ Available regular hours .

- The fraction of time for direct patient-care (which is the fraction of time a nurse is on duty excluding leaves, orientation and education), is given by

$\frac{\text { Avalable Hours }}{N \text { of nurses } x \text { nb of shifts per nurse per schedule } x n \text { bo hours per shift }}=$ Fraction of Time for Direct patient care

- The used FTE over the six-week scheduling period, is given by

$$
\frac{\text { Used hours }}{\text { Nbof hours per shift } x \text { Nb of shifts per schedule Fraction of direct patient care }}=\text { Used FTE }
$$

- The overtime in FTE, is given by 


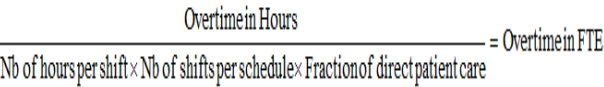

- The required resources are assessed as follows:

Budgeted NHPPD $\times$ Census $\times 42=$ Required Hours.

$$
\frac{\text { Required Hours }}{\text { Nb of hours per shift } x \text { Nb of shifts per schedule } x \text { Fraction of direct patient care }}=\text { Required FTE }
$$

- The staffing shortage level is given by:

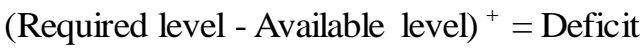

$$
\text { g) }
$$

- The overstaffing level is given by

(Required level - Available level) $^{-}=$Overstaffing $^{-}$

- The surplus level (level of nurses used above the require ments) is given by:

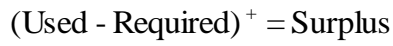

- The level of shortage (the level of nurses below the require ments) is given by:

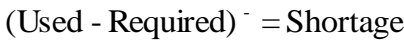

In the above notations, we used the following:

$(\mathrm{x})^{+}=\max (\mathrm{x}, 0)$ and $(\mathrm{x})^{-}=\max (-\mathrm{x}, 0)$

Based on the above relationships, it follows that the available number of hours is of 215038 and hence the fraction of time for direct patient care is $73 \%$. From equation (3), the used FTE is of 1354 nurse and from (4) the overtime (OT) is of 240 FTE nurse. Consequently, the total practiced deficit was of 232 FTE nurse. The surplus level is of 67 FTE nurses and the shortage level is of 42 FTE nurses. On the other hand, the nursing allocation over the period of interest resulted in an overstaffing of 17 FTE nurses leading to a net deficit of 215 FTE nurse. This result is significantly different from the initial hospital estimate of 300 FTE nurse. Compared to the existing number of nurses, the net deficit represents $16 \%$.

\section{DISCUSSION AND ANALYSIS}

Each nurse spends an average of $\mathbf{7 3 \%}$ of her time for direct-patient care (accounting for leaves, education and orientation). The number of used nurses is of 1354 FTE leading to an OT of 240 FTE. The required staffing level to cover the budgeted NHPPD is of 1329 FTE. Some nursing units are understaffed while others are overstaffed. The aggregate understaffing level is 232 FTE while the overstaffing level of $\mathbf{1 7}$ FTE, resulting in a staffing deficit of 215 FTE. A surplus of 67 FTE is used above the requirement defined by the budgeted NHPPD and the census, resulting on a potential saving of $\mathbf{2 8 \%}$. Some units are however using less FTEsthan requiredyielding an aggregate shortage of 42 FTE. Finally, the situation at the aggregate level (of the 32 units) is summarized in the following table:

Table (1). Summary of the main results

\begin{tabular}{|c|c|}
\hline Performance Indicator & Result \\
\hline Available & $\mathbf{1 1 1 4}$ FTE \\
\hline Required & $\mathbf{1 3 2 9}$ FTE \\
\hline Staffing deficit & $\mathbf{2 1 5}$ FTE (16\%) \\
\hline Surplus level & $\mathbf{6 7}$ FTE \\
\hline Surplus as a percent of Total OT & $\mathbf{2 8 \%}$ \\
\hline
\end{tabular}

In particular, instead of 300 FTEs, the analysis shows that 215 FTEs should be enough to cover the staffing shortage for the 32 in-patient units. This says that the current staffing shortage in the nursing staff is of $16 \%$. The analys is extends to show that 67 FTE represent a surplus in nurses' allocation (exceeding the approved budgeted NHPPD) for the six-week schedule examined in the study, resulting in $28 \%$ surplus in OT resources. This number looks significant and may present a potential opportunity for savings in OT.

Figure 1 displays the current staffing situation in terms of available, required, and used staffing levels at each of the 32 in-patient units of the hospital. From the figure, it is clear that in most cases the available number of nurses is smaller than requiredleading to a deficit. Overstaffing is also practiced.

For instance, one can observe that for U26 unit the number of used nurses exceeds the required level. It is even worse at the U1 and U18 units where the numbers of used nurses exceed the numbers of available nurses which in turn exceed the numbers of required nurses. On the other hand, shortage is 
observed at several units. Amazingly, at U29 the number of used nurses is even smaller than the number of available nurses which in turn is smaller than the required level. That is, the nurses of the units are perhaps being floated to other units while U29 itself suffers from severe shortage. It is clear therefore that the staffing policies are not optimized in a way to minimize both surpluses and shortages.

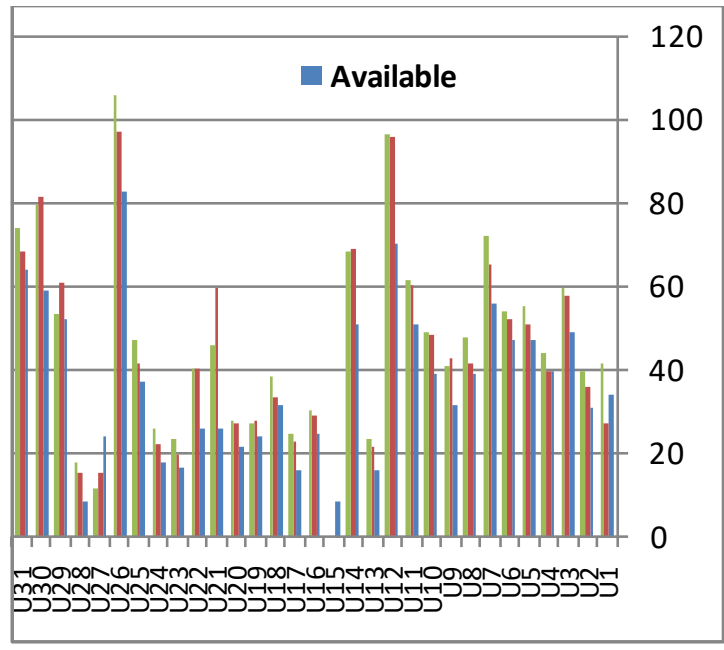

Figure 1: Available, required, and used staffing levels at each unit

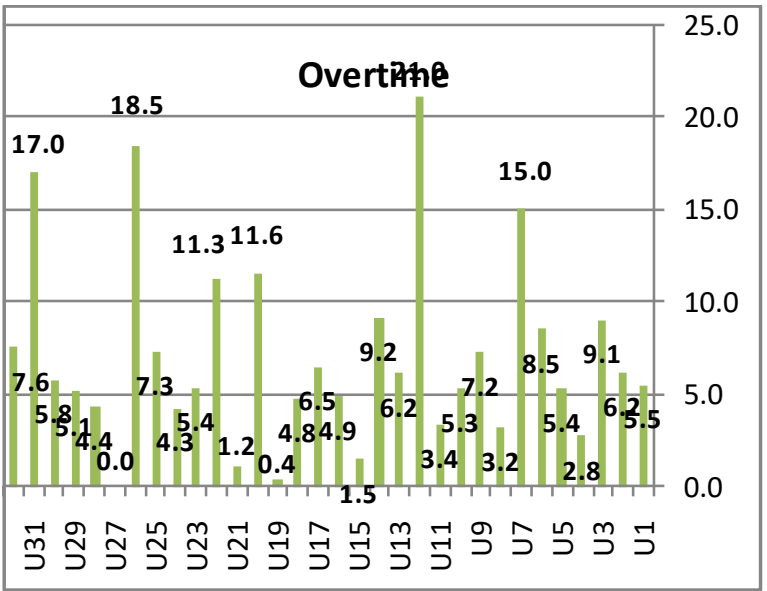

Figure 2: Overtime in all 32-units

Figure 2 displays overtime in all 32 units. Except for U27 unit, all the remaining 31 units opt for overtime. Most of the units use a level below 10 FTE nurses. Extreme cases are found at U12, U26,
U31 and U7 units with $21,18.5,17$, and 15 FTE nurses, respectively.

Figure 3 exhibits the staffing deficit in all the units. Figure 5-4 exhibits the staffing deficit in percent of the required level.Four (out of 32) units are not understaffed. The highest deficit level is as large as 23.8 FTE nurses (observed at U12 unit). However, the highest deficit as a percent of the required level is observed at the U15 and U27 units with $100 \%$ deficit, followed by the U29 unit with $43 \%$ deficit.

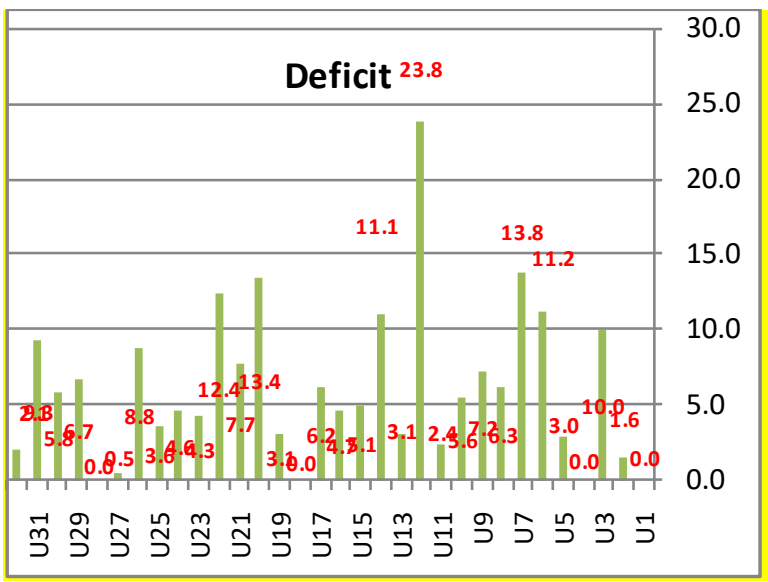

Figure 3: Staffing deficit per unit

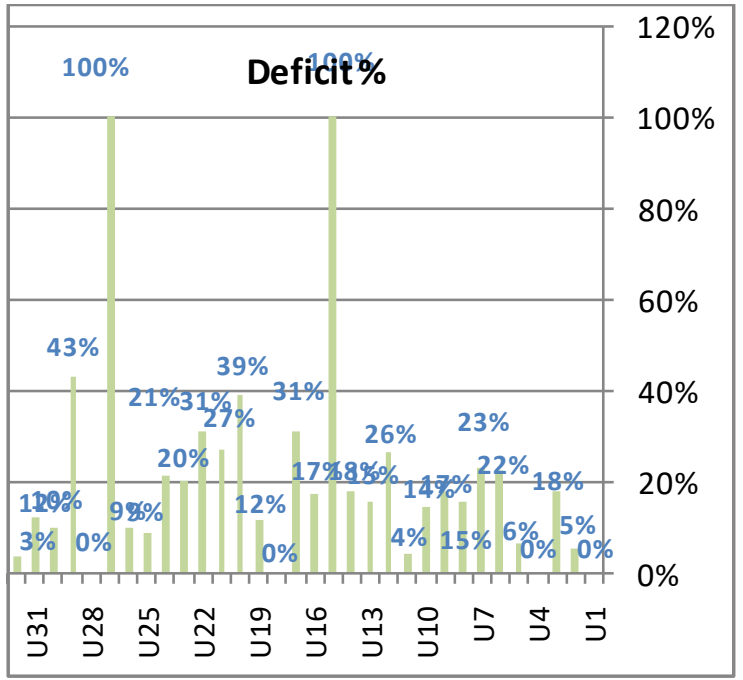

Figure 5-4: Staffing deficit in percent of the required level 
Figure 5 and Figure 6 exhibit the surplus per unit and the distribution of surplus over all units, respectively.

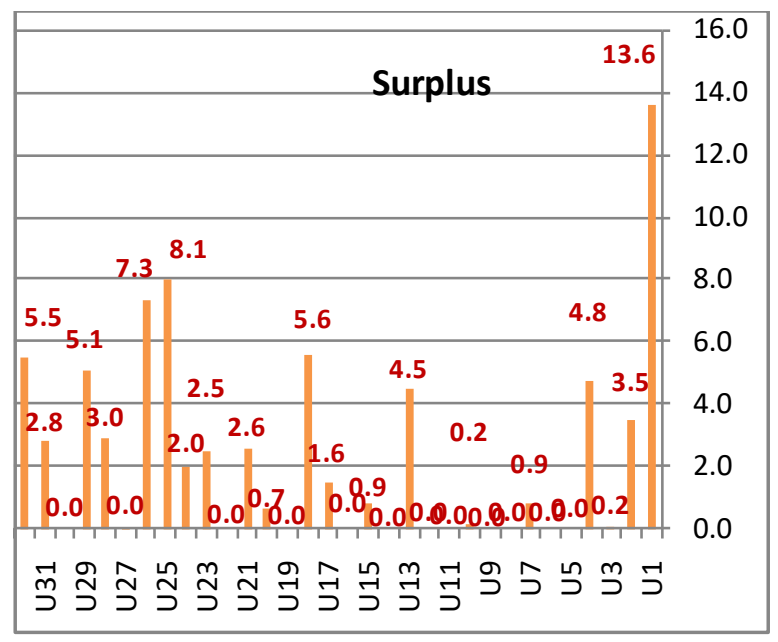

Figure 5: Surplus at each unit

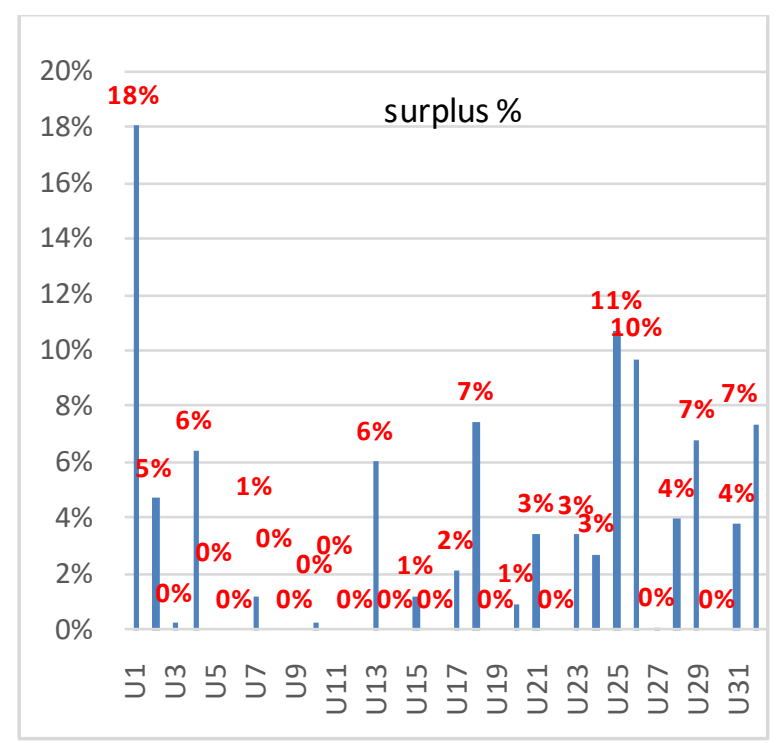

Figure 6: Surplus as a percent of the available staffing level at each unit

Clearly, surplus is not very common and is practiced in a relatively small number of units, in which and according to the nursing department some special care is required. It is however unusual to observe 12.8 FTE nurses of surplus at a single unit in the case where a hospital severely suffers from major understaffing.
Concerning the expected impact of the findings of this study, one important question may arise as whether the current $28 \%$ surplus may be transformed into future saving if more sophisticated staffing policies may be generated. The nursing department argues against it and attempts to justify this surplus level through the following arguments:

- The current patient-care acuity seems to be not reflecting the real hospital situation and consequently the real need for patient care may exceed the estimated requirements.

- Some units may use more nurses than initially planned for. This is due to the fluctuations in patients conditions.

- Some special units may use some very special care to some special patients exceeding the regular require ments.

However, it is not sure how much each of the above arguments is responsible for among the $28 \%$ surplus. Work in progress (Louly et al. [16]) investigates the real reasons for the surplus for a larger horizon of scheduling time. The initial results prove indeed some strong potential for major savings. In fact, the authors show that a large fraction of this surplus turns out to be avoidable through appropriate planning and scheduling techniques.

\section{CONCLUSIONS}

This study attempts to investigate to what extent efficiency was used in the current staffing practices, given the high level of staffing shortage at a large hospital (the largest in the Gulf region). The results show that the right staffing shortage level is of 215 FTE, or equivalently $16 \%$ of the required level. This number is significantly below the 300 FTE level estimated by the nursing department. Moreover, the analysis demonstrated that overtime exceeded the approved NHPPD by $28 \%$ for the investigated six-week period. Given the huge budget of about $\$ 11.5$ million allocated for overtime, this $28 \%$ surplus requires further investigation and analysis both to validate it and to interpret it without excluding the potential for future saving.

Work in progress (Louly et al. [16]) extends the current study to a larger horizon to examine the validity of the $28 \%$ surplus. The study also isolates the candidate factors contributing to this surplus (e.g., inadequate matching between current applied NHPPD and real demand in patient care, variability in the acuity of patient-care, special services provided 
by the hospitals, deliberate reduction in efficiency to increase nurses' satisfaction, etc.). The study shows that the $28 \%$ surplus is still valid over a one-year horizon. Moreover, the initial results prove that most of the surplus is avoidable through appropriate staffing and scheduling.

\section{ACKNOWLEDGEMENT}

This paper is supported by the National Plan for Science \& Technology (NPST) program at King Saud University (project number MAT-1491).

\section{REFERENCES}

[1]. Aiken, L. H., Clarke, S. P., Sloane, D. M., Sochalski, J., \& Silber, J. H. (2002). Hospital nurse staffing and patient mortality, nurse burnout, and job dissatisfaction. JAMA: the journal of the American Medical Association,288(16), 1987-1993.

[2]. Mchugh, M. D., Shang, J., Sloane, D. M., \& Aiken, L. H. (2011). Risk factors for hospitalacquired 'poor glycemic control': a casecontrol study. International Journal for Quality in Health Care, 23(1), 44-51.

[3]. Schwab, F., Meyer, E., Geffers, C., \& Gastmeier, P. (2012). Understaffing, overcrowding, inappropriate nurse: ventilated patient ratio and nosocomial infections: which parameter is the best reflection of deficits?. Journal of Hospital Infection, 80(2), 133-139.

[4]. Van den Heede, K., Sermeus, W., Diya, L., Clarke, S. P., Lesaffre, E., Vleugels, A., \& Aiken, L. H. (2009). Nurse staffing and patient outcomes in Belgian acute hospitals: crosssectional analysis of administrative data. International Journal of Nursing Studies, 46(7), 928-939.

[5]. Needleman, J., Buerhaus, P., Mattke, S., Stewart, M., \& Zelevinsky, K. (2002). Nursestaffing levels and the quality of care in hospitals. New England Journal of Medicine, 346(22), 1715-1722..

[6]. Tubbs-Cooley, H. L., Cimiotti, J. P., Silber, J. H., Sloane, D. M., \& Aiken, L. H. (2013). An observational study of nurse staffing ratios and hospital readmission among children admitted for common conditions. BMJ quality \& safety.

[7]. McGahan, M., Kucharski, G., \& Coyer, F. (2012). Nurse staffing levels and the incidence of mortality and morbidity in the adult intensive care unit: A literature review. Australian Critical Care, 25(2), 64-77.
[8]. Akinci, F., \& Krolikowski, D. (2005). Nurse staffing levels and quality of care in Northeastern Pennsylvania nursing homes. Applied Nursing Research, 18(3), 130137.

[9]. Ball, J. E., Murrells, T., Rafferty, A. M., Morrow, E., \& Griffiths, P. (2013). 'Care left undone'during nursing shifts: associations with workload and perceived quality of care. BMJ quality \& safety.

[10]. Kalisch, B. J., Tschannen, D., \& Lee, K. H. (2011). Do staffing levels predict missed nursing care?. International Journal for Quality in Health Care, 23(3), 302-308.

[11]. Clarke, S. P. (2007). Making the business case for nursing: Justifying investments in nurse staffing and high-quality practice environments. Nurse Leader, 5(4), 34-38.

[12]. Savitz, L. A., Jones, C. B., \& Bernard, S. (2005). Quality indicators sensitive to nurse staffing in acute care settings. Agency for Healthcare Research and Quality (US)

[13]. Goryakin, Y., Griffiths, P., \& Maben, J. (2011). Economic evaluation of nurse staffing and nurse substitution in health care: a scoping review. International Journal of Nursing Studies, 48(4), 501-512.

[14]. Azaiez, M. N., \& Al Sharif, S. S. (2005). A 01 goal programming model for nurse scheduling. Computers \& Operations Research, 32(3), 491-507.

[15]. Maenhout, B., \& Vanhoucke, M. (2013). An integrated nurse staffing and scheduling analysis for longer-term nursing staff allocation problems. Omega,41(2), 485-499.

[16]. Louly, M., Gharbi, A. , A zaiez, M. N., A. Bouras, A. (2014). A New Aggregate Planning Approach for Nurse Staffing: Application to a Large Hospital in the Gulf Region. Under review. 